\title{
Proposal of a two-qutrit contextuality test free of the finite precision and compatibility loopholes
}

\author{
Adán Cabelld* \\ Departamento de Física Aplicada II, Universidad de Sevilla, E-41012 Sevilla, Spain \\ Marcelo Terra Cunha \\ Departamento de Matemática, Universidade Federal de Minas Gerais, \\ Caixa Postal 702, 30123-970, Belo Horizonte, MG, Brazil
}

(Dated: October 29, 2018)

\begin{abstract}
It has been argued that any test of quantum contextuality is nullified by the fact that perfect orthogonality and perfect compatibility cannot be achieved in finite precision experiments. We introduce experimentally testable two-qutrit violations of inequalities for noncontextual theories in which compatibility is guaranteed by the fact that measurements are performed on separated qutrits. The inequalities are inspired by the basic building block of the Kochen-Specker proof of quantum contextuality for a qutrit, despite the fact that their proof is completely independent of it.
\end{abstract}

PACS numbers: 03.65.Ud, 03.65.Ta, 03.67.Mn, 42.50.Xa

Introduction - Contextuality is a fascinating property of nature: The result of an experiment may depend on other compatible experiments that may be performed. This is surprising because the experiment gives the same result when repeated after any number of compatible experiments. It is also surprising because the probability of obtaining any particular result does not depend on which compatible experiments might be performed (probabilities are noncontextual). Nature's contextuality was pointed out by the discovery that some predictions of quantum mechanics (QM) cannot be reproduced by any noncontextual theory, and that this conflict occurs for any state of any system with three or more distinguishable states [1]. Quantum contextuality has far-reaching consequences, such as the impossibility of describing nature by classical theories with bounded speed of information [2] or bounded density of memory [3], or the possibility of device-independent eternally secure communications [4].

The original demonstration by Kochen and Specker (KS) 1] is usually considered a tour de force because it is based on a 117-vertex graph. However, this graph has an 8-vertex building block, which we refer to as the basic diagram (Fig. 1). In a KS graph, each vertex is seen as a yes or no proposition and linked vertices are compatible propositions where no more than one of them can be assigned the yes answer (a property sometimes called exclusiveness). The original 117-vertex graph was built to give a state-independent proof of the impossibility of assigning noncontextual answers reproducing the predictions of quantum mechanics for a three-level system (like a spin 1). Accordingly, a triangle represents a set of three compatible propositions, one of them receiving the yes answer (given quantum mechanically by a projective complete measurement, like the $z$ component of a spin 1 particle). The basic building block is also referred to as a yes-no diagram, in the sense that if the answer yes is noncontextually assigned to vertex $i$, any valid assignment must give to the vertex $f$ the value no. This Letter was motivated by this basic diagram, as we will show below.

In 1999, a debate on the physical impact and experimental testability of the Kochen-Specker theorem started in the pages of Physical Review Letters. Meyer [5] and Kent 6] pointed out that finite precision measurement nullifies the physical content of the Kochen-Specker theorem. More than 20 papers have been published since then supporting (for instance, 7, 8]) or criticizing (for instance, 9, 10]) this conclusion. More recently, a series of experimental tests with ions [1], neutrons [12], photons [13, 14], and nuclear magnetic resonance systems 15] have been questioned due to a variant of the objection of Meyer and Kent called the compatibility loophole [11, 16].

In this Letter we present a proposal for a type of experiment that (we hope) will definitely close the debate on the physical relevance and experimental testability of the Kochen-Specker theorem and stimulate a new generation of experiments.

Since it is impossible to have contextuality for a twolevel system, subjected to projective measurements, the three-state quantum system, or qutrit, is considered the simplest example of contextuality. We give here a statedependent quantum contextuality demonstration [17, 18] with a twofold objective: to provide a clear example for the nonspecialized reader and to fix notation to be used in the two-qutrit case. Suppose the qutrit is initially in the state

$$
|i\rangle=\frac{1}{\sqrt{3}}(|0\rangle+|1\rangle+|2\rangle),
$$

where $|0\rangle,|1\rangle$, and $|2\rangle$ are three orthogonal states. Then the measurement represented by the projector $|f\rangle\langle f|$, 
with

$$
|f\rangle=\frac{1}{\sqrt{3}}(|0\rangle-|1\rangle+|2\rangle)
$$

gives the result 1 (representing "yes"), with (quantum mechanical) probability

$$
|\langle f \mid i\rangle|^{2}=\frac{1}{9} .
$$

This already establishes the yes-yes possibility and we need only to prove the existence of the six other vertices obeying all compatibility and exclusiveness properties of the KS basic building block. For this, consider the observable

$$
T_{0}=a_{0}\left|a_{0}\right\rangle\left\langle a_{0}\left|+b_{0}\right| b_{0}\right\rangle\left\langle b_{0}\left|+c_{0}\right| c_{0}\right\rangle\left\langle c_{0}\right|,
$$

where $\left|a_{0}\right\rangle,\left|b_{0}\right\rangle$, and $\left|c_{0}\right\rangle$ are the following orthogonal states:

$$
\begin{aligned}
& \left|a_{0}\right\rangle=\frac{1}{\sqrt{2}}(|1\rangle-|2\rangle), \\
& \left|b_{0}\right\rangle=\frac{1}{\sqrt{2}}(|1\rangle+|2\rangle), \\
& \left|c_{0}\right\rangle=|0\rangle .
\end{aligned}
$$

Then, according to QM, the result $a_{0}$ can never be obtained since $\left\langle a_{0} \mid i\right\rangle=0$. In addition, in any noncontextual theory in agreement with $\mathrm{QM}$, the result of $T_{0}$ cannot be $b_{0}$ since $\left\langle f \mid b_{0}\right\rangle=0$. Therefore, in any noncontextual theory in agreement with QM, the initial state $|i\rangle$ together with the positive probability (3) imply $T_{0}=c_{0}$, whenever the yes answer should be found for $|f\rangle$. Consider also

$$
T_{1}=a_{1}\left|a_{1}\right\rangle\left\langle a_{1}\left|+b_{1}\right| b_{1}\right\rangle\left\langle b_{1}\left|+c_{1}\right| c_{1}\right\rangle\left\langle c_{1}\right|,
$$

where

$$
\begin{aligned}
\left|a_{1}\right\rangle & =\frac{1}{\sqrt{2}}(|0\rangle-|1\rangle), \\
\left|b_{1}\right\rangle & =\frac{1}{\sqrt{2}}(|0\rangle+|1\rangle), \\
\left|c_{1}\right\rangle & =|2\rangle .
\end{aligned}
$$

The same reasoning leads to the conclusion that, in any noncontextual theory in agreement with $\mathrm{QM}, T_{1}=c_{1}$ whenever $f$ is to happen.

However, $T_{0}=c_{0}$ and $T_{1}=c_{1}$ cannot happen simultaneously since $\left\langle c_{1} \mid c_{0}\right\rangle=0$. Therefore, there is no noncontextual assignment of results to the six propositions $\left|a_{0}\right\rangle\left\langle a_{0}|, \ldots,| c_{1}\right\rangle\left\langle c_{1}\right|$ in agreement with the predictions of QM for a qutrit prepared in the state $|i\rangle$ and postselected in the state $|f\rangle$. Indeed, the orthogonality relations between these eight states constitutes the building block of the Kochen-Specker proof [1, 19]. These eight quantum states obey all orthogonality conditions represented on the building block diagram (see Fig. 1), but
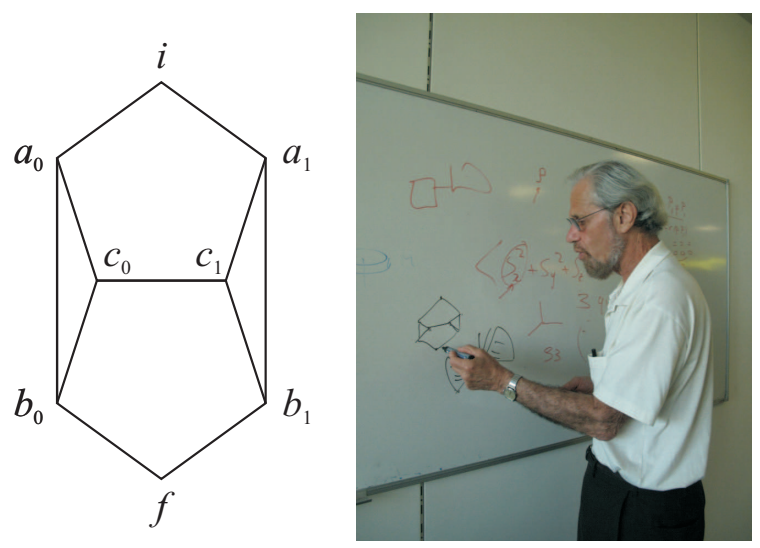

FIG. 1. Building block of the Kochen-Specker proof of quantum contextuality (left). Vertices represent propositions; two of them are joined when the propositions are compatible and both cannot be true. Kochen drawing the block in Zürich in 2009 (right).

the nonorthogonality of $|i\rangle$ and $|f\rangle$ allows for a yes-yes answer, ruled out by noncontextual assignments.

What if perfect orthogonality cannot be experimentally achieved? Then the previous argument vanishes. For instance, the block of Fig. 1 cannot be constructed if the unit vectors are restricted to vectors with rational components [5] or to a set where each vector participates only of one context 7], although in both cases these sets are dense in the set of unit vectors and therefore are undistinguishable from the set of all unit vectors by experiments with finite precision [5 10]. A related problem affecting some recent experimental violations [11 13] of noncontextual inequalities [20, 21] involving sequential measurements on the same physical system is the fact that these inequalities are based on the assumption that the sequential measurements are perfectly compatible, something which does not occur in actual experiments, so to conclude contextuality extra assumptions are needed [11, 16].

Here we avoid these extra assumptions by measuring one of the observables in one qutrit and the second observable on a distant qutrit. Spatial separation provides a physical basis to the assumption that both measurements are not only approximately but perfectly compatible. The combination of state-independent KochenSpecker proofs and correlated systems is a standard way to show contextuality and nonlocality for composite systems [17, 22, 24, 25]. Here, instead, we do not require a state-independent proof. Our central result will be to adapt the previous one-qutrit example to find twoqutrit quantum violations for noncontextual inequalities derived under the same inspiration.

For this purpose we start by deriving an inequality valid for any realistic theory (i.e., those assigning predefined answers for all possible questions) based on the observables of the block of Kochen and Specker and in- 
volving correlations between measurements on two different parties $A$ and $B$.

Lemma 1. - Consider $D_{j}$ as dichotomic observables (with possible outcomes 1 and 0 ), $T_{j}$ as trichotomic observables (with possible outcomes $a_{j}, b_{j}$, and $c_{j}$ ), with $j=0,1$ and labels $A$ and $B$ corresponding to the respective party. Then, for any realistic theory, the following inequality holds:

$$
\mathcal{K} \leq 0
$$

where

$$
\begin{aligned}
\mathcal{K}= & \sum_{j \neq k=0}^{1} P\left(D_{j}^{A}=1, D_{k}^{B}=1\right)-P\left(D_{0}^{A}=1, T_{k}^{B}=a_{k}\right) \\
& -P\left(T_{j}^{A}=a_{j}, D_{0}^{B}=1\right)-P\left(D_{1}^{A}=1, T_{k}^{B}=b_{k}\right) \\
& -P\left(T_{j}^{A}=b_{j}, D_{1}^{B}=1\right)-P\left(T_{j}^{A}=a_{j}, T_{k}^{B}=b_{k}\right) \\
& -P\left(T_{j}^{A}=a_{j}, T_{j}^{B}=b_{j}\right)-P\left(T_{j}^{A}=b_{j}, T_{j}^{B}=a_{j}\right) \\
& -P\left(T_{0}^{A}=a_{0}, T_{0}^{B}=c_{0}\right)-P\left(T_{0}^{A}=c_{0}, T_{0}^{B}=a_{0}\right) \\
& -P\left(T_{0}^{A}=b_{0}, T_{0}^{B}=c_{0}\right)-P\left(T_{0}^{A}=c_{0}, T_{0}^{B}=b_{0}\right) \\
& -P\left(T_{j}^{A}=c_{j}, T_{k}^{B}=c_{k}\right),
\end{aligned}
$$

where $P\left(D_{0}^{A}=1, T_{0}^{B}=a_{0}\right)$ denotes the joint probability of obtaining the results 1 and $a_{0}$ for $D_{0}^{A}$ and $T_{0}^{B}$, respectively.

Proof.-Each probability in (18) can be written as a sum of a set of probabilities for complete hidden-variable states. In our scenario there are four dichotomic observables, $D_{0}^{A}, D_{1}^{A}, D_{0}^{B}$, and $D_{1}^{B}$, and four trichotomic observables, $T_{0}^{A}, T_{1}^{A}, T_{0}^{B}$, and $T_{1}^{B}$. The proof is a bookkeeping exercise showing that each term that adds to the positive term $P\left(D_{j}^{A}=1, D_{k}^{B}=1\right)$ also appears (with the right multiplicity) at some of the negative terms, implying $\mathcal{K} \leq 0$. A detailed calculation is given in the supplemental material ??.

Notice that, despite the fact that the inequality (18) is inspired by the KS diagram applied to a pair of threelevel systems in a singlet state, as will be made clear by Eqs. (13), it is independent of it in the sense that the proof is not based on a diagram, and even a computer programmed to search for realistic inequalities could have found it.

It is also important to stress that inequality (18) also holds for any local theory. This means that, when the choice of measurement in one of the particles is spacelike separated from the result of the measurement on the other particle, then (18) is a KS inspired Bell inequality.

In translating the KS basic diagram into four realistic questions, exclusiveness of each triangle was used, but the noncontextual assumption deserves more. For each part, we also have the following logical implications:

$$
\begin{aligned}
& T_{j}=a_{j} \Rightarrow D_{0}=0, \\
& T_{j}=b_{j} \Rightarrow D_{1}=0, \\
& T_{j}=c_{j} \Rightarrow T_{k} \neq c_{k}(j \neq k) .
\end{aligned}
$$

A noncontextual assignment is given by answers to the four questions following the conditions (21). Only 14 out of the 36 realistic states are noncontextual. With fewer allowed states, more restrictions can be obtained. One can now prove the following.

Lemma 2. - Consider $D_{j}$ and $T_{j}$ as in Lemma 1. Moreover, consider that these questions obey, for each party, conditions (21). The following inequality holds:

$$
\begin{aligned}
P_{\mathrm{nc}}\left(D_{0}^{A}=1, D_{1}^{B}=1\right) & -P_{\mathrm{nc}}\left(D_{0}^{A}=1, T_{0}^{B}=a_{0}\right) \\
& -P_{\mathrm{nc}}\left(D_{0}^{A}=1, T_{1}^{B}=a_{1}\right) \leq 0 .
\end{aligned}
$$

Where $P_{\mathrm{nc}}$ is used to remind that only noncontextual hidden-variable states are taken into account.

Proof.-Again, it is simple bookkeeping showing that all terms which contribute to the positive term also appear at (at least one) negative ones. Details and other inequalities are provided in the supplemental material 26].

Inequalities (23) are tight, with respect to noncontextual states, and can be violated by realistic states which do not obey some of the relations (21). In this sense, even with an experiment prepared with spacelike separations between $A$ and $B$, they should not be considered as Bell inequalities. Their violation rules out a different set of hidden-variable models: noncontextual ones. From the technical point of view, that is a great advantage, since an experimentalist need not bother with guaranteeing spatial-like separations.

With the same kind of proof one can deduce the following.

Lemma 3.-With the same conditions of Lemma 2,

$$
\begin{aligned}
P_{\mathrm{nc}}\left(D_{0}^{A}=1, D_{1}^{B}=1\right) & -\sum_{j} P_{\mathrm{nc}}\left(T_{j}^{A}=b_{j}, T_{j}^{B}=a_{j}\right) \\
& -\sum_{j \neq k} P_{\mathrm{nc}}\left(T_{j}^{A}=c_{j}, T_{k}^{B}=c_{k}\right) \leq 0 .
\end{aligned}
$$

And also other similar expressions. Again, (24) is tight with respect to noncontextual states, and can be violated with realistic contextual ones.

Quantum violation.-The same quantum system will violate all inequalities shown here. It is just a two particle generalization of the previous described state-dependent proof of impossibility of noncontextual models for quantum mechanics. Consider a two-qutrit system prepared in the state

$$
|\psi\rangle=\frac{1}{\sqrt{3}}(|02\rangle-|11\rangle+|20\rangle),
$$


and the observables

$$
\begin{aligned}
& D_{0}^{A}=D_{1}^{B}=|i\rangle\langle i|, \\
& D_{1}^{A}=D_{0}^{B}=|f\rangle\langle f|, \\
& T_{0}^{A}=a_{0}\left|a_{0}\right\rangle\left\langle a_{0}\left|+b_{0}\right| b_{0}\right\rangle\left\langle b_{0}\left|+c_{0}\right| c_{0}\right\rangle\left\langle c_{0}\right|, \\
& T_{0}^{B}=a_{0}\left|b_{1}\right\rangle\left\langle b_{1}\left|+b_{0}\right| a_{1}\right\rangle\left\langle a_{1}\left|+c_{0}\right| c_{1}\right\rangle\left\langle c_{1}\right|, \\
& T_{1}^{A}=a_{1}\left|a_{1}\right\rangle\left\langle a_{1}\left|+b_{1}\right| b_{1}\right\rangle\left\langle b_{1}\left|+c_{1}\right| c_{1}\right\rangle\left\langle c_{1}\right|, \\
& T_{1}^{B}=a_{1}\left|b_{0}\right\rangle\left\langle b_{0}\left|+b_{1}\right| a_{0}\right\rangle\left\langle a_{0}\left|+c_{1}\right| c_{0}\right\rangle\left\langle c_{0}\right|,
\end{aligned}
$$

with $|i\rangle,|f\rangle,\left|a_{j}\right\rangle,\left|b_{j}\right\rangle$, and $\left|c_{j}\right\rangle$ as defined in Eqs (11), (2), (5), and (7). Then the predictions of QM are

$$
P\left(D_{j}^{A}=1, D_{k}^{B}=1\right)=\frac{1}{27}
$$

where $j, k \in\{0,1\}$ and $j \neq k$, and the remaining probabilities in (8b) are null. Therefore, according to QM,

$$
\mathcal{K}_{\mathrm{QM}}=\frac{2}{27} \approx 0.074
$$

violating inequality (18), and inequalities (23) and (24) read

$$
\frac{1}{27} \leq 0
$$

Discussion. - Inequality (18) was deduced using a translation of the basic KS diagram into a set of four propositions. After this translation is done, its validity rests only on hidden-variable hypothesis of a preexisting reality which is reveled by measurements. Inequalities (23) and (24) are based on these four questions and on the constraints (21) given by noncontextual hypothesis. Because the lemmas were written and proved, there is no necessity of going back to the diagrams, despite the fact that their essence is captured by those relations.

In all these inequalities, each term involves pairs of compatible questions, asked for different parts of the composed system. Moreover, being a quantitative statement, the argument is not sensible to finite precision problems, given that precision is enough to make violations meaningful. In this sense those are proposals for quantum violations of noncontextuality free from compatibility and finite precision loopholes.

Testing the violation of inequality (18) is very demanding experimentally. It requires testing 22 joint probabilities and, assuming that one obtains $P\left(D_{j}^{A}=1, D_{k}^{B}=\right.$ $1) \approx \frac{1}{27}$ and $\epsilon$ for the other 20 probabilities, one must have $\epsilon<\frac{1}{270} \approx 0.0037$ in order to observe a violation of inequality (18). The importance of this result is that it explicitly shows that there is no fundamental obstacle observing Kochen-Specker contextuality on (pairs of) qutrits. No additional assumptions are needed to deal with the fact that measurements have a finite precision and compatibility between sequential measurements is not perfect.
Inequalities (23) are less demanding experimentally. Indeed, they can be translated as conditional probabilities giving inequalities like

$$
\begin{aligned}
P_{\mathrm{nc}}\left(D_{1}^{A}=1 \mid D_{0}^{B}=1\right) & -P_{\mathrm{nc}}\left(T_{0}^{A}=a_{0} \mid D_{0}^{B}=1\right) \\
& -P_{\mathrm{nc}}\left(T_{1}^{A}=a_{1} \mid D_{0}^{B}=1\right) \leq 0,
\end{aligned}
$$

which gives larger violations, since QM predicts $P\left(D_{1}^{A}=\right.$ $\left.1 \mid D_{0}^{B}=1\right)=\frac{1}{9}$, allowing for $\epsilon^{\prime}<\frac{1}{27} \approx 0.037$. Another interesting point is that inequality (25) only involves one question and one answer in part $B: D_{0}^{B}=1$, and an experiment would only involve three possible measurements in part $A$, with coincidence countings of each of these three with one and the same condition in $B$. It is also stressed in the supplementary material 26] how much this situation resembles the first example discussed in this Letter.

In this Letter we have established experimentally testable contextual inequalities, based on the building block of Kochen-Specker demonstration of the impossibility of assigning noncontextual answers to all possible predictions of quantum mechanics. We hope that this proposal will definitely close the debate on the physical relevance and experimental testability of the Kochen-Specker theorem, and induce a new generation of loophole-free experiments.

This work was stimulated by a discussion with A. Zeilinger. The final work benefited from a discussion with D. Cavalcanti. A.C. acknowledges support from Capes and ICEx (Belo Horizonte, Brazil), the Spanish MICINN Project No. FIS2008-05596, and the WennerGren Foundation. M.T.C. acknowledges support from Brazilian CNPq, Fapemig, and INCT-IQ.

* adan@us.es

[1] S. Kochen and E. P. Specker, J. Math. Mech. 17, 59 (1967).

[2] J. S. Bell, Physics (Long Island City, N.Y.) 1, 195 (1964).

[3] M. Kleinmann et al., arXiv:1007.3650.

[4] K. Horodecki et al., arXiv:1006.0468.

[5] D. A. Meyer, Phys. Rev. Lett. 83, 3751 (1999).

[6] A. Kent, Phys. Rev. Lett. 83, 3755 (1999).

[7] R. Clifton and A. Kent, Proc. R. Soc. London, Ser. A 456, 2101 (2000).

[8] J. Barrett and A. Kent, Stud. Hist. Philos. Mod. Phys. 35, 151 (2004).

[9] N. D. Mermin, quant-ph/9912081.

[10] A. Cabello, Phys. Rev. A 65, 052101 (2002).

[11] G. Kirchmair et al., Nature (London) 460, 494 (2009).

[12] H. Bartosik et al., Phys. Rev. Lett. 103, 040403 (2009).

[13] E. Amselem et al., Phys. Rev. Lett. 103, 160405 (2009).

[14] B. H. Liu et al., Phys. Rev. A 80, 044101 (2009).

[15] O. Moussa et al., Phys. Rev. Lett. 104, 160501 (2010).

[16] O. Gühne et al., Phys. Rev. A 81, 022121 (2010).

[17] A. Stairs, Phil. Sci. 50, 578 (1983). 
[18] R. Clifton, Am. J. Phys. 61, 443 (1993); H. Bechmann Johansen, Am. J. Phys. 62, 471 (1994); P. E. Vermaas, Am. J. Phys. 62, 658 (1994).

[19] S. Kochen and E. P. Specker, in Symposium on the Theory of Models, edited by J. W. Addison et al., (NorthHolland, Amsterdam, Holland, 1965), p. 177.

[20] A. Cabello, Phys. Rev. Lett. 101, 210401 (2008).

[21] P. Badzia̧g et al., Phys. Rev. Lett. 103, 050401 (2009).

[22] S. Kochen, to A. Shimony (private communication), reported in [17], p. 581, and in [23], p. 481.

[23] P. Heywood and M. L. G. Redhead, Found. Phys. 13, 481 (1983).

[24] A. Cabello, Phys. Rev. Lett. 104, 220401 (2010).

[25] L. Aolita, R. Gallego, A. Acín, A. Chiuri, G. Vallone, P. Mataloni, and A. Cabello (to be published).

[26] See supplemental material below for the proofs of the lemmas, some generalizations, and technical discussion of the results.

\section{SUPPLEMENTARY MATERIAL}

We start by proving the first Lemma stated in the Letter.

Lemma 1.-Consider $D_{j}$ as dichotomic observables (with possible outcomes 1 and 0 ), $T_{j}$ as trichotomic observables (with possible outcomes $a_{j}, b_{j}$, and $c_{j}$ ), with $j=0,1$ and labels $A$ and $B$ corresponding to the respective party. Then, for any realistic theory, the following inequality holds:

$$
\mathcal{K} \leq 0
$$

where

$$
\begin{aligned}
\mathcal{K}= & \sum_{j \neq k=0}^{1} P\left(D_{j}^{A}=1, D_{k}^{B}=1\right)-P\left(D_{0}^{A}=1, T_{k}^{B}=a_{k}\right) \\
& -P\left(T_{j}^{A}=a_{j}, D_{0}^{B}=1\right)-P\left(D_{1}^{A}=1, T_{k}^{B}=b_{k}\right) \\
& -P\left(T_{j}^{A}=b_{j}, D_{1}^{B}=1\right)-P\left(T_{j}^{A}=a_{j}, T_{k}^{B}=b_{k}\right) \\
& -P\left(T_{j}^{A}=a_{j}, T_{j}^{B}=b_{j}\right)-P\left(T_{j}^{A}=b_{j}, T_{j}^{B}=a_{j}\right) \\
& -P\left(T_{0}^{A}=a_{0}, T_{0}^{B}=c_{0}\right)-P\left(T_{0}^{A}=c_{0}, T_{0}^{B}=a_{0}\right) \\
& -P\left(T_{0}^{A}=b_{0}, T_{0}^{B}=c_{0}\right)-P\left(T_{0}^{A}=c_{0}, T_{0}^{B}=b_{0}\right) \\
& -P\left(T_{j}^{A}=c_{j}, T_{k}^{B}=c_{k}\right),
\end{aligned}
$$

where, $P\left(D_{0}^{A}=1, T_{0}^{B}=a_{0}\right)$ denotes the joint probability of obtaining the results 1 and $a_{0}$ for $D_{0}^{A}$ and $T_{0}^{B}$, respectively.

Proof.-Each probability in (18) can be written as a sum of a set of probabilities for complete hidden variable states. In our scenario there are four dichotomic observables, $D_{0}^{A}, D_{1}^{A}, D_{0}^{B}$, and $D_{1}^{B}$, and four trichotomic observables, $T_{0}^{A}, T_{1}^{A}, T_{0}^{B}$, and $T_{1}^{B}$. The proof is a bookkeeping exercise showing that each term that adds to the positive term $P\left(D_{j}^{A}=1, D_{k}^{B}=1\right)$ also appears (with the right multiplicity) at some of the negative terms, implying $\mathcal{K} \leq 0$. We will need some notation. A complete hidden variable state will be given by

$$
\left(d_{0}^{A}, d_{1}^{A}, t_{0}^{A}, t_{1}^{A}, d_{0}^{B}, d_{1}^{B}, t_{0}^{B}, t_{1}^{B}\right) .
$$

Any summation without defined index denotes summation over all the unspecified symbols. With this convention, the positive contributions for $\mathcal{K}$ are given by

$$
\begin{aligned}
\sum_{j \neq k=0}^{1} P\left(D_{j}^{A}=\right. & \left.1, D_{k}^{B}=1\right) \\
= & \sum P\left(1, d_{1}^{A}, t_{0}^{A}, t_{1}^{A}, d_{0}^{B}, 1, t_{0}^{B}, t_{1}^{B}\right) \\
& +\sum P\left(d_{0}^{A}, 1, t_{0}^{A}, t_{1}^{A}, 1, d_{1}^{B}, t_{0}^{B}, t_{1}^{B}\right) .
\end{aligned}
$$

namely, $2 \times 2^{2} 3^{4}=648$ terms, where $3^{4}=81$ appear with multiplicity two. Let us begin by chasing all terms $\left(1, d_{1}^{A}, t_{0}^{A}, t_{1}^{A}, d_{0}^{B}, 1, t_{0}^{B}, t_{1}^{B}\right)$. The first negative term will cancel all terms like $\left(1, d_{1}^{A}, t_{0}^{A}, t_{1}^{A}, d_{0}^{B}, 1, a_{0}, t_{1}^{B}\right)$ and $\left(1, d_{1}^{A}, t_{0}^{A}, t_{1}^{A}, d_{0}^{B}, 1, t_{0}^{B}, a_{1}\right)$, so one eliminates all terms with $t_{0}^{B}=a_{0}$ or $t_{1}^{B}=a_{1}$. It remains to be canceled all terms such that the pair $\left(t_{0}^{B}, t_{1}^{B}\right)$ be given by $\left(b_{0}, b_{1}\right),\left(b_{0}, c_{1}\right),\left(c_{0}, b_{1}\right)$, and $\left(c_{0}, c_{1}\right)$. The term $P\left(T_{j}^{A}=b_{j}, D_{1}^{B}=1\right)$ cancels terms where $t_{0}^{A}=$ $b_{0}$ or $t_{1}^{A}=b_{1}$. But we still have to look for the 64 terms $\left(1, d_{1}^{A}, a_{0} / c_{0}, a_{1} / c_{1}, d_{0}^{B}, 1, b_{0} / c_{0}, b_{1} / c_{1}\right)$. The term $P\left(T_{j}^{A}=a_{j}, T_{k}^{B}=b_{k}\right)$, with $j=0$, cancels all remaining terms with $t_{0}^{A}=a_{0}$, except those with $t_{1}^{B}=c_{1}$. Some of these terms are included in $P\left(T_{0}^{A}=a_{0}, T_{0}^{B}=c_{0}\right)$, and we are now concerned only with the terms $\left(1, d_{1}^{A}, a_{0}, a_{1} / c_{1}, d_{0}^{B}, 1, b_{0}, c_{1}\right)$ and $\left(1, d_{1}^{A}, c_{0}, a_{1} / c_{1}, d_{0}^{B}, 1, b_{0} / c_{0}, b_{1} / c_{1}\right)$. On the same way, but with $j=1, t_{1}^{A}=a_{1}$ with $t_{0}^{B}=b_{0}$ goes away, leaving $\left(1, d_{1}^{A}, a_{0}, c_{1}, d_{0}^{B}, 1, b_{0}, c_{1}\right), \quad\left(1, d_{1}^{A}, c_{0}, a_{1}, d_{0}^{B}, 1, c_{0}, b_{1} / c_{1}\right)$, and $\left(1, d_{1}^{A}, c_{0}, c_{1}, d_{0}^{B}, 1, b_{0}, b_{1} / c_{1}\right)$. The first of these terms goes with $P\left(T_{j}^{A}=a_{j}, T_{j}^{B}=b_{j}\right)$, the last goes with $P\left(T_{0}^{A}=c_{0}, T_{0}^{B}=b_{0}\right)$, while the remaining reduces to $\left(1, d_{1}^{A}, c_{0}, a_{1}, d_{0}^{B}, 1, c_{0}, b_{1}\right)$, due to $P\left(T_{j}^{A}=c_{j}, T_{k}^{B}=c_{k}\right)$, which is finally canceled by $P\left(T_{j}^{A}=a_{j}, T_{j}^{B}=b_{j}\right)$.

Now we can follow a similar strategy for the terms $\quad\left(d_{0}^{A}, 1, t_{0}^{A}, t_{1}^{A}, 1, d_{1}^{B}, t_{0}^{B}, t_{1}^{B}\right)$. Starting from $P\left(T_{j}^{A}=a_{j}, D_{0}^{B}=1\right)$, which eliminates all terms with $t_{0}^{A}=a_{0}$ or $t_{1}^{A}=a_{1}$, and $P\left(D_{i}^{A}=1, T_{k}^{B}=b_{k}\right)$, which eliminates $t_{0}^{B}=b_{0}$ and $t_{1}^{B}=b_{1}$, we are left with $\left(d_{0}^{A}, 1, b_{0} / c_{0}, b_{1} / c_{1}, 1, d_{1}^{B}, a_{0} / c_{0}, a_{1} / c_{1}\right)$. The term $P\left(T_{j}^{A}=b_{j}, T_{j}^{B}=a_{j}\right)$, with $j=0$, reduces the task to $\left(d_{0}^{A}, 1, b_{0}, b_{1} / c_{1}, 1, d_{1}^{B}, c_{0}, a_{1} / c_{1}\right)$ and $\left(d_{0}^{A}, 1, c_{0}, b_{1} / c_{1}, 1, d_{1}^{B}, a_{0} / c_{0}, a_{1} / c_{1}\right)$. The same term, with $j=1$, reduces even more to $\left(d_{0}^{A}, 1, b_{0}, b_{1}, 1, d_{1}^{B}, c_{0}, c_{1}\right), \quad\left(d_{0}^{A}, 1, b_{0}, c_{1}, 1, d_{1}^{B}, c_{0}, a_{1} / c_{1}\right)$, $\left(d_{0}^{A}, 1, c_{0}, b_{1}, 1, d_{1}^{B}, a_{0} / c_{0}, c_{1}\right), \quad$ and $\left(d_{0}^{A}, 1, c_{0}, c_{1}, 1, d_{1}^{B}, a_{0} / c_{0}, a_{1} / c_{1}\right)$. The two first terms are eliminated by $P\left(T_{0}^{A}=b_{0}, T_{0}^{B}=c_{0}\right)$, 
while $P\left(T_{0}^{A}=c_{0}, T_{0}^{B}=a_{0}\right)$ reduces the order two to $\left(d_{0}^{A}, 1, c_{0}, b_{1}, 1, d_{1}^{B}, c_{0}, c_{1}\right)$, and $\left(d_{0}^{A}, 1, c_{0}, c_{1}, 1, d_{1}^{B}, c_{0}, a_{1} / c_{1}\right)$. The term $P\left(T_{j}^{A}=\right.$ $\left.c_{j}, T_{k}^{B}=c_{k}\right)$ with $j=0$ eliminates the first and reduces the second to $\left(d_{0}^{A}, 1, c_{0}, c_{1}, 1, d_{1}^{B}, c_{0}, a_{1}\right)$, which is eliminated by the same term with $j=1$.

As already said, 81 terms appear with twice each in the positive part. We close the demonstration by proving that they also appear (at least) twice in the negative contributions. They are $\left(1,1, t_{0}^{A}, t_{1}^{A}, 1,1, t_{0}^{B}, t_{1}^{B}\right)$. Let us start by the terms $\left(1,1, a_{0}, t_{1}^{A}, 1,1, t_{0}^{B}, t_{1}^{B}\right)$. They appear once at $P\left(T_{j}^{A}=a_{j}, D_{0}^{B}=1\right)$, for $j=0$, and a second time at $P\left(D_{0}^{A}=1, T_{k}^{B}=a_{k}\right), k=0$, if $t_{0}^{B}=a_{0}$, at $P\left(T_{j}^{A}=a_{j}, T_{j}^{B}=b_{j}\right), j=0$, if $t_{0}^{B}=b_{0}$, and at $P\left(T_{0}^{A}=a_{0}, T_{0}^{B}=c_{0}\right)$, in case $t_{0}^{B}-c_{0}$. Analogously, for the terms $\left(1,1, b_{0}, t_{1}^{A}, 1,1, t_{0}^{B}, t_{1}^{B}\right)$, they appear once at $P\left(T_{j}^{A}=b_{j}, D_{1}^{B}=1\right)$, and again at $P\left(T_{j}^{A}=b_{j}, T_{j}^{B}=a_{j}\right)$, $j=0$, if $t_{0}^{B}=a_{0}$, at $P\left(D_{1}^{A}=1, T_{k}^{B}=b_{k}\right), k=0$, if $t_{0}^{B}=b_{0}$, and at $P\left(T_{0}^{A}=b_{0}, T_{0}^{B}=c_{0}\right)$, if $t_{0}^{B}=c_{0}$. Finally, for the states $\left(1,1, c_{0}, t_{1}^{A}, 1,1, t_{0}^{A}, t_{1}^{A}\right)$, they appear once at $P\left(D_{0}^{A}=1, T_{k}^{B}=a_{k}\right), k=1$, if $t_{1}^{B}=a_{1}$, at $P\left(D_{1}^{A}=1, T_{k}^{B}=b_{k}\right), k=1$, if $t_{1}^{B}=b_{1}$, and at $P\left(T_{j}^{A}=c_{j}, T_{k}^{B}=c_{k}\right), k=1$, if $t_{1}^{B}=c_{1}$. The second appearance can be noted at $P\left(T_{0}^{A}=c_{0}, T_{0}^{B}=a_{0}\right)$, if $t_{0}^{B}=a_{0}$, at $P\left(T_{0}^{A}=c_{0}, T_{0}^{B}=b_{0}\right)$ if $t_{0}^{B}=b_{0}$, while for $t_{0}^{B}=c_{0}$ we split again into three terms: $P\left(T_{j}^{A}, D_{0}^{B}\right)$, $j=1$ for $t_{1}^{A}=a_{1}, P\left(T_{j}^{A}=b_{j}, D_{1}^{B}=1\right), j=1$, for $t_{1}^{A}=b_{1}$, and $P\left(T_{j}^{A}=c_{j}, T_{k}^{B}=c_{k}\right), j=1$, for $t_{1}^{A}=c_{1}$.

Before proving Lemma 2, let us list all noncontextual hidden-variable states giving answers to the four questions which one can ask for one party, subjected to the restrictions:

$$
\begin{aligned}
& T_{j}=a_{j} \Rightarrow D_{0}=0, \\
& T_{j}=b_{j} \Rightarrow D_{1}=0, \\
& T_{j}=c_{j} \Rightarrow T_{k} \neq c_{k}(j \neq k) .
\end{aligned}
$$

As stated, only the following 14 out of the 36 realistic states are noncontextual:

$$
\left(0, q_{1}, a_{0}, a_{1}\right),\left(0,0, a_{0}, b_{1}\right),\left(0, q_{1}, a_{0}, c_{1}\right),\left(0,0, b_{0}, a_{1}\right),
$$$$
\left(q_{0}, 0, b_{0}, b_{1}\right),\left(q_{0}, 0, b_{0}, c_{1}\right),\left(0, q_{1}, c_{0}, a_{1}\right),\left(q_{0}, 0, c_{0}, b_{1}\right) \text {. }
$$

When considering two parties, the cross product must be applied, resulting in $14^{2}=196$ noncontextual states.

We are now ready to state and prove a generalized version of Lemma 2 in the text.

Lemma 2'.- Consider $D_{j}$ and $T_{j}$ as in Lemma 1. Moreover, consider these questions to obey, for each party, conditions (21). The following inequalities hold:

$$
\begin{aligned}
P_{\mathrm{nc}}\left(D_{0}^{A}=1, D_{1}^{B}=1\right) & -P_{\mathrm{nc}}\left(D_{0}^{A}=1, T_{0}^{B}=a_{0}\right) \\
& -P_{\mathrm{nc}}\left(D_{0}^{A}=1, T_{1}^{B}=a_{1}\right) \leq 0, \\
P_{\mathrm{nc}}\left(D_{1}^{A}=1, D_{0}^{B}=1\right) & -P_{\mathrm{nc}}\left(T_{0}^{A}=a_{0}, D_{0}^{B}=1\right) \\
& -P_{\mathrm{nc}}\left(T_{1}^{A}=a_{1}, D_{0}^{B}=1\right) \leq 0, \\
& -P_{\mathrm{nc}}\left(D_{0}^{A}=1, T_{0}^{B}=b_{0}\right) \\
& -P_{\mathrm{nc}}\left(D_{0}^{A}=1, T_{1}^{B}=b_{1}\right) \leq 0, \\
& \\
P_{\mathrm{nc}}\left(D_{0}^{A}=1, D_{1}^{B}=1\right) & -P_{\mathrm{nc}}\left(T_{0}^{A}=b_{0}, D_{0}^{B}=1\right) \\
& -P_{\mathrm{nc}}\left(T_{1}^{A}=b_{1}, D_{0}^{B}=1\right) \leq 0 .
\end{aligned}
$$

Where $P_{\mathrm{nc}}$ is used to remind that only noncontextual hidden-variable states are taken into account.

Proof. - Let us prove inequality (23a) in detail. The others are analogous. Note that only three one-party states allow for $D_{0}^{A}=1:\left(1,0, b_{0}, b_{1}\right),\left(1,0, b_{0}, c_{1}\right)$, and $\left(1,0, c_{0}, b_{1}\right)$. In the same way, only three states of party $B$ will allow for $D_{1}^{B}=1$ : $\left(0,1, a_{0}, a_{1}\right),\left(0,1, a_{0}, c_{1}\right)$, and $\left(0,1, c_{0}, a_{1}\right)$. So we have 9 noncontextual states contributing for $P_{\mathrm{nc}}\left(D_{0}^{A}=1, D_{1}^{B}=1\right)$. But note that six of these states have $t_{0}^{B}=a_{0}$, and the other three have $t_{1}^{B}=a_{1}$, so once again all terms which add for the positive part also appear in the negative one. As probabilities are non-negative, the resulting inequality applies.

To see that inequality (23a) is tight, just use state $\left(1,0, b_{0}, b_{1}, 0,1, a_{0}, c_{1}\right)$, while $\left(1,0, b_{0}, b_{1}, 0,1, a_{0}, a_{1}\right)$ is a realistic contextual state (in the sense that it is an assignment of values which does not obey the assumption of noncontextuality) which violates it.

It is now time to prove the following.

Lemma 3.-With the same conditions of Lemma 2,

$$
\begin{aligned}
P_{\mathrm{nc}}\left(D_{0}^{A}=1, D_{1}^{B}=1\right) & -\sum_{j} P_{\mathrm{nc}}\left(T_{j}^{A}=b_{j}, T_{j}^{B}=a_{j}\right) \\
& -\sum_{j \neq k} P_{\mathrm{nc}}\left(T_{j}^{A}=c_{j}, T_{k}^{B}=c_{k}\right) \leq 0 .
\end{aligned}
$$

Proof. - It is again necessary to cancel out the same nine terms which contribute to $P_{\mathrm{nc}}\left(D_{0}^{A}=1, D_{1}^{B}=1\right)$. Four of them have $t_{0}^{A}=b_{0}$ and $t_{0}^{B}=a_{0}$. Also four of them have $t_{1}^{A}=b_{1}$ and $t_{1}^{B}=a_{1}$, but one was taken twice. The remaining two terms are canceled by the second summation term, since one has $\left(T_{0}^{A}=c_{0}, T_{1}^{B}=c_{1}\right)$ and the other $\left(T_{1}^{A}=c_{1}, T_{0}^{B}=c_{0}\right)$.

Again, (24) is tight with respect to noncontextual states, as the state $\left(1,0, b_{0}, c_{1}, 0,1, a_{0}, c_{1}\right)$ shows, and can be violated with realistic contextual ones like $\left(1,0, a_{0}, a_{1}, 0,1, b_{0}, b_{1}\right)$. 
Quantum violation.-Now let us discuss in more details how the quantum violation of the presented inequalities is directly related to the first example discussed in the Letter. For this, we will focus on the inequality

$$
\begin{aligned}
P_{\mathrm{nc}}\left(D_{1}^{A}=1 \mid D_{0}^{B}=1\right) & -P_{\mathrm{nc}}\left(T_{0}^{A}=a_{0} \mid D_{0}^{B}=1\right) \\
& -P_{\mathrm{nc}}\left(T_{1}^{A}=a_{1} \mid D_{0}^{B}=1\right) \leq 0 .
\end{aligned}
$$

Since every term is conditioned to $D_{0}^{B}=1$, we can consider a projective measurement on part $B$ with the result corresponding to $|f\rangle\langle f|$ appearing. Since the conditional state of

$$
|\psi\rangle=\frac{1}{\sqrt{3}}(|02\rangle-|11\rangle+|20\rangle)
$$

given that $|f\rangle_{B}$ was obtained is $|i\rangle_{A}$, one is faced with the quantum probabilities

$$
\begin{aligned}
& P\left(D_{1}^{A}=1 \mid D_{0}^{B}=1\right)=|\langle f \mid i\rangle|^{2}=\frac{1}{9}, \\
& P\left(T_{0}^{A}=a_{0} \mid D_{0}^{B}=1\right)=\left|\left\langle a_{0} \mid i\right\rangle\right|^{2}=0, \\
& P\left(T_{1}^{A}=a_{1} \mid D_{0}^{B}=1\right)=\left|\left\langle a_{1} \mid i\right\rangle\right|^{2}=0,
\end{aligned}
$$

from where not only the violation is evident, but also the relation with the previous discussed example must be clear. 